\title{
APPROXIMATION OF OPTIMAL CONTROL PROBLEMS IN THE COEFFICIENT FOR THE $p$-LAPLACE EQUATION. I. CONVERGENCE RESULT*
}

\author{
EDUARDO CASAS ${ }^{\dagger}$, PETER I. KOGUT KAND GÜNTER LEUGERING $^{\S}$
}

\begin{abstract}
We study a Dirichlet optimal control problem for a quasi-linear monotone elliptic equation, the so-called weighted $p$-Laplace problem. The coefficient of the $p$-Laplacian, the weight $u$, we take as a control in $B V(\Omega) \cap L^{\infty}(\Omega)$. In this article, we use box-type constraints for the control such that there is a strictly positive lower and some upper bound. In order to handle the inherent degeneracy of the $p$-Laplacian, we use a regularization, sometimes referred to as the $\varepsilon$ - $p$-Laplacian. We derive existence and uniqueness of solutions to the underlying boundary value problem and the optimal control problem. In fact, we introduce a two-parameter model for the weighted $\varepsilon$ - $p$ Laplacian, where we approximate the nonlinearity by a bounded monotone function, parametrized by $k$. Further, we discuss the asymptotic behavior of the solutions to the regularized problem on each $(\varepsilon, k)$-level as the parameters tend to zero and infinity, respectively.
\end{abstract}

Key words. nonlinear Dirichlet problem, optimal control, control in coefficients

AMS subject classifications. 35J70, 49J20, 49J45, 93C73

DOI. $10.1137 / 15 \mathrm{M} 1028108$

1. Introduction. Control in the coefficients of elliptic problems has a long history of its own, starting with the work of Murat [10, 11] and Tartar [14]. The constrained optimal control problem (OCP) in the coefficients of the leading order differential expressions was first discussed in detail by Casas [2] in the case of the classical Laplace equation, where the scalar coefficient $u$ in the $\operatorname{div}(u \nabla \cdot)$ formulation was taken as control satisfying box constraints with a strictly positive lower and some upper bound together with a slope constraint. The problem of existence and uniqueness of the underlying boundary value problem and the corresponding OCP was treated, and an optimality system has been derived and analyzed. Analogous results for the case of general quasi-linear elliptic equations of the type $\operatorname{div}(a(u, \nabla \cdot))$ remained open. In this article we treat the case of the weighted $p$-Laplacian, where $a(u, \nabla y)=u|\nabla y|^{p-2} \nabla y$. The corresponding quasi-linear differential operator, $-\operatorname{div}\left(u|\nabla y|^{p-2} \nabla y\right)$, in principle, has degeneracies as $\nabla y$ tends to zero and also if $u$ approaches zero. Moreover, when the term $u|\nabla y|^{p-2}$ is regarded as the coefficient of the Laplace operator, we also have the case of unbounded coefficients. In order to avoid degeneracy with respect to the control $u$, we assume that $u$ is bounded away from zero. For the precise statements, see the next section. We leave the case of potentially degenerating controls to a future contribution. Instead, in this article, we focus on the degeneracies related to the nonlinearity. A number of regularizations have been suggested in the literature.

\footnotetext{
${ }^{*}$ Received by the editors June 29, 2015; accepted for publication (in revised form) February 18, 2016; published electronically June 1, 2016.

http://www.siam.org/journals/sicon/54-3/M102810.html

$\dagger$ †epartmento Matemática Aplicada y Ciencias de la Computación, E.T.S.I.I. y T., Universidad de Cantabria, 39005 Santander, Spain (eduardo.casas@unican.es).

${ }_{\ddagger}^{\ddagger}$ Department of Differential Equations, Dnipropetrovsk National University, 49010 Dnipropetrovsk, Ukraine (p.kogut@i.ua).

$\S$ Chair of Applied Mathematics II, University of Erlangen-Nuremberg, D-91058 Erlangen, Germany (guenter.leugering@fau.de). This author's research was supported by the DFG-EC315 "Engineering of Advanced Materials" and by the Spanish Ministerio de Economía y Competitividad under projects MTM2011-22711 and MTM2014-57531-P.
} 
See [12] for a discussion for what has come to be known as the $\varepsilon$-p-Laplace problem, i.e., $\Delta_{u, \varepsilon, p} y:=\operatorname{div}\left(u\left(\varepsilon+|\nabla y|^{2}\right)^{\frac{p-2}{2}}\right) \nabla y$. While the $\varepsilon$-p-Laplacian regularizes the degeneracy as the gradients tend to zero, the term $u|\nabla y|^{p-2}$, viewed again as a coefficient for the otherwise linear problem, may grow large. Therefore, we introduce yet another regularization that leads to a sequence of monotone and bounded approximation $\mathcal{F}_{k}\left(|\nabla y|^{2}\right)$ of $|\nabla y|^{2}$. For fixed parameter $p \in[2, \infty)$, and control $u$, we arrive at a two-parameter problem governed by

$$
\Delta_{\varepsilon, k, p} y-y:=\operatorname{div}\left(u\left(\varepsilon+\mathcal{F}_{k}\left(|\nabla y|^{2}\right)\right)^{\frac{p-2}{2}}\right) \nabla y-y .
$$

Finally, we have to deal with a two-parameter family of OCPs in the coefficients for monotone nonlinear differential equations. We consequently provide the wellposedness analysis for the underlying partial differential equations as well as for the OCPs. After that we pass to the limits as $k \rightarrow \infty$ and $\varepsilon \rightarrow 0$. The approximations and regularizations are considered to be useful not only for the mathematical analysis, but also for the purpose of numerical simulations.

An important point, arising after the solvability of the optimization problem, is the question of optimality conditions. The classical approach to deriving such conditions is based on the Lagrange principle. However, in the case when the control is considered as a scalar coefficient of the weighted $p$-Laplacian, the classical adjoint system often cannot be directly constructed due to the lack of differential properties of the solution to the boundary value problem with respect to control variables. To overcome this difficulty, in the forthcoming second part of this paper, we derived the optimality conditions passing to the limit in optimality conditions for a two-parameter family of approximating control problems.

2. Setting of the OCP. Let $\Omega$ be a bounded open subset of $\mathbb{R}^{N}(N \geq 1)$ with a Lipschitz boundary. Let $p$ be a real number such that $2 \leq p<\infty$. By $B V(\Omega)$ we denote the space of all functions in $L^{1}(\Omega)$ for which the norm

$$
\begin{aligned}
\|f\|_{B V(\Omega)} & =\|f\|_{L^{1}(\Omega)}+\int_{\Omega}|D f| \\
& =\|f\|_{L^{1}(\Omega)}+\sup \left\{\int_{\Omega} f \operatorname{div} \varphi d x: \varphi \in C_{0}^{1}\left(\Omega ; \mathbb{R}^{N}\right),|\varphi(x)| \leq 1 \text { for } x \in \Omega\right\}
\end{aligned}
$$

is finite.

Let $\xi_{1}, \xi_{2}$ be given elements of $L^{\infty}(\Omega) \cap B V(\Omega)$ satisfying the conditions

$$
0<\alpha \leq \xi_{1}(x) \leq \xi_{2}(x) \text { a.e. in } \Omega,
$$

where $\alpha$ is a given positive value. Let $z_{d} \in L^{2}(\Omega)$ and $f \in L^{2}(\Omega)$ be given distributions. The OCP we consider in this paper is to minimize the discrepancy between the distribution $z_{d} \in L^{2}(\Omega)$ and the solutions of the boundary value problem

$$
\begin{gathered}
-\Delta_{p}(u, y)+y=f \quad \text { in } \Omega, \\
y=0 \text { on } \partial \Omega
\end{gathered}
$$

by choosing an appropriate weight function $u \in \mathfrak{A}_{a d}$ as control. Here, $\Delta_{p}(u, \cdot)$ is the generalized $p$-Laplacian, i.e.,

$$
\Delta_{p}(u, y)=\operatorname{div}\left(u(x)|\nabla y|^{p-2} \nabla y\right), \quad \text { where }|\nabla y|^{p-2}:=|\nabla y|_{\mathbb{R}^{N}}^{p-2}=\left(\sum_{i=1}^{N}\left|\frac{\partial y}{\partial x_{i}}\right|^{2}\right)^{\frac{p-2}{2}}
$$


and the class of admissible $B V$-controls $\mathfrak{A}_{a d}$ is defined as follows:

$$
\mathfrak{A}_{a d}=\left\{u \in B V(\Omega) \mid \xi_{1}(x) \leq u(x) \leq \xi_{2}(x) \text { a.e. in } \Omega\right\} .
$$

It is clear that $\mathfrak{A}_{a d}$ is a nonempty convex subset of $L^{1}(\Omega)$ with an empty topological interior.

More precisely, we are concerned with the following OCP:

$$
\text { Minimize }\left\{I(u, y)=\int_{\Omega}\left|y-z_{d}\right|^{2} d x+\int_{\Omega}|D u|\right\}
$$

subject to the constraints $(2.2)-(2.4)$.

As usual, a function $y \in W_{0}^{1, p}(\Omega)$ is said to be a solution of $(2.2)-(2.3)$ if

$$
\int_{\Omega} u|\nabla y|^{p-2}(\nabla y, \nabla \varphi)_{\mathbb{R}^{N}} d x+\int_{\Omega} y \varphi d x=\int_{\Omega} f \varphi d x \quad \forall \varphi \in W_{0}^{1, p}(\Omega) .
$$

The existence of a unique solution to the boundary value problem (2.2)-(2.3) follows from an abstract theorem on monotone operators; see, for instance, [9] or [13, section II.2].

Theorem 2.1. Let $V$ be a reflexive separable Banach space. Let $V^{*}$ be the dual space, and let $A: V \rightarrow V^{*}$ be a bounded, semicontinuous, coercive, and strictly monotone operator. Then the equation $A y=f$ has a unique solution for each $f \in V^{*}$. Moreover, Ay $=f$ if and only if $\langle A \varphi, \varphi-y\rangle \geq\langle f, \varphi-y\rangle$ for all $\varphi \in V^{*}$.

Here, the above mentioned properties of the strict monotonicity, semicontinuity, and coercivity of the operator $A$ have, respectively, the following meaning:

$$
\begin{aligned}
& \langle A y-A v, y-v\rangle_{V^{*} ; V} \geq 0 \quad \forall y, v \in V ; \\
& \langle A y-A v, y-v\rangle_{V^{*} ; V}=0 \Longrightarrow y=v ;
\end{aligned}
$$

the function $t \mapsto\langle A(y+t v), w\rangle_{V^{*} ; V}$ is continuous $\forall y, v, w \in V$;

$$
\lim _{\|y\|_{V} \rightarrow \infty} \frac{\langle A y, y\rangle_{V^{*} ; V}}{\|y\|_{V}}=+\infty .
$$

In our case, we can define the operator $A$ as a mapping $W_{0}^{1, p}(\Omega) \rightarrow W^{-1, q}(\Omega)$ by

$$
\langle A \varphi, v\rangle_{W^{-1, q}(\Omega) ; W_{0}^{1, p}(\Omega)}:=\int_{\Omega} u(x)|\nabla \varphi|^{p-2}(\nabla \varphi, \nabla v)_{\mathbb{R}^{N}} d x+\int_{\Omega} v \varphi d x .
$$

Then it is easy to show that $A y=-\Delta_{p}(u, y)+y$ and $A$ satisfies all assumptions of Theorem 2.1 (for the details, we refer the reader to $[9,12]$ ). As a consequence of this theorem, we also know that $y \in W_{0}^{1, p}(\Omega)$ satisfies (2.6) if and only if the Minty relation holds, i.e., if

$$
\int_{\Omega} u|\nabla \varphi|^{p-2}(\nabla \varphi, \nabla \varphi-\nabla y)_{\mathbb{R}^{N}} d x+\int_{\Omega} \varphi(\varphi-y) d x \geq \int_{\Omega} f(\varphi-y) d x \quad \forall \varphi \in W_{0}^{1, p}(\Omega) .
$$

Taking this into account, the set of admissible pairs to problem (2.2)-(2.5),

(2.12) $\Xi=\left\{(u, y) \mid u \in \mathfrak{A}_{a d}, y \in W_{0}^{1, p}(\Omega),(u, y)\right.$ are related by equality (2.6) $\}$,

is nonempty. With this notation, the control problem (2.1)-(2.5) can be written as follows:

$$
\text { (P) } \min _{(u, y) \in \Xi} I(u, y) \text {. }
$$


3. Existence of optimal solutions. In this section we focus on the solvability of OCP $(\mathrm{P})$. Hereinafter, we suppose that the space $B V(\Omega) \times W_{0}^{1, p}(\Omega)$ is endowed with the norm $\|(u, y)\|_{B V(\Omega) \times W_{0}^{1, p}(\Omega)}:=\|u\|_{B V(\Omega)}+\|y\|_{W_{0}^{1, p}(\Omega)}$. Moreover, $q$ will denote the conjugate of $p: q=\frac{p}{p-1}$.

Remark 3.1. We recall that a sequence $\left\{f_{k}\right\}_{k=1}^{\infty}$ converges weakly* to $f$ in $B V(\Omega)$ if and only if the following two conditions hold (see [1]): $f_{k} \rightarrow f$ strongly in $L^{1}(\Omega)$ and $D f_{k} \stackrel{*}{\rightarrow} D f$ weakly* in the space of Radon measures $\mathcal{M}\left(\Omega ; \mathbb{R}^{N}\right)$. Moreover, if

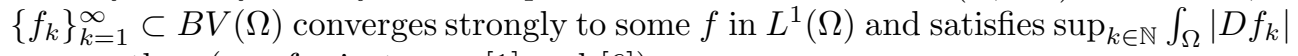
$<+\infty$, then (see, for instance, [1] and [6])

$$
\begin{aligned}
& \text { (i) } f \in B V(\Omega) \text { and } \int_{\Omega}|D f| \leq \liminf _{k \rightarrow \infty} \int_{\Omega}\left|D f_{k}\right| \text {; } \\
& \text { (ii) } f_{k} \stackrel{*}{\rightarrow} f \text { in } B V(\Omega) \text {. }
\end{aligned}
$$

As an obvious consequence of these notions, we have the following property.

Lemma 3.2. Let $\left\{\left(u_{k}, y_{k}\right)\right\}_{k \in \mathbb{N}} \subset \Xi$ be a sequence such that $u_{k} \stackrel{*}{\rightarrow} u$ in $B V(\Omega)$ and $y_{k} \rightarrow y$ in $W_{0}^{1, p}(\Omega)$. Then we have

$$
\lim _{k \rightarrow \infty} \int_{\Omega}\left(\nabla y_{k}, \nabla \varphi\right)_{\mathbb{R}^{N}} u_{k} d x=\int_{\Omega}(\nabla y, \nabla \varphi)_{\mathbb{R}^{N}} u d x \quad \forall \varphi \in C_{0}^{\infty}(\Omega) .
$$

Proof. Since $u_{k} \rightarrow u$ in $L^{1}(\Omega)$ and $\left\{u_{k}\right\}_{k \in \mathbb{N}}$ is bounded in $L^{\infty}(\Omega)$, we infer $u_{k} \rightarrow u$ strongly in $L^{r}(\Omega)$ for every $1 \leq r<+\infty$. In particular, $u_{k} \rightarrow u$ in $L^{q}(\Omega)$ and $\left(\nabla y_{k}, \nabla \varphi\right)_{\mathbb{R}^{N}} \rightarrow(\nabla y, \nabla \varphi)_{\mathbb{R}^{N}}$ in $L^{p}(\Omega)$. Hence, it is immediate to pass to the limit and to deduce (3.2).

As a consequence, we have the following property.

Corollary 3.3. Let $\left\{\left(u_{k}, y_{k}\right)\right\}_{k \in \mathbb{N}} \subset \Xi$ and $\left\{\zeta_{k}\right\}_{k \in \mathbb{N}} \subset W_{0}^{1, q}(\Omega)$ be sequences such that $u_{k} \stackrel{*}{\rightarrow} u$ in $B V(\Omega), y_{k} \rightarrow y$ in $W_{0}^{1, p}(\Omega)$, and $\zeta_{k} \rightarrow \zeta$ in $W_{0}^{1, q}(\Omega)$. Then

$$
\lim _{k \rightarrow \infty} \int_{\Omega}\left(\nabla y_{k}, \nabla \zeta_{k}\right)_{\mathbb{R}^{N}} u_{k} d x=\int_{\Omega}(\nabla y, \nabla \zeta)_{\mathbb{R}^{N}} u d x .
$$

Our next step concerns the study of topological properties of the set of admissible solutions $\Xi$ to problem $(2.2)-(2.5)$.

The following result is crucial for our further analysis.

TheOREm 3.4. Let $\left\{\left(u_{k}, y_{k}\right)\right\}_{k \in \mathbb{N}} \subset \Xi$ be a bounded sequence. Then, there is a pair $(u, y) \in \Xi$ such that, up to a subsequence, $u_{k} \stackrel{*}{\rightarrow} u$ in $B V(\Omega)$ and $y_{k} \rightarrow y$ in $W_{0}^{1, p}(\Omega)$.

Proof. By Remark 3.1 and compactness properties of $B V(\Omega) \times W_{0}^{1, p}(\Omega)$, there exist a subsequence of $\left\{\left(u_{k}, y_{k}\right)\right\}_{k \in \mathbb{N}}$, still denoted by the same indices, and functions $u \in B V(\Omega)$ and $y \in W_{0}^{1, p}(\Omega)$ such that

$$
u_{k} \stackrel{*}{\rightarrow} u \text { in } B V(\Omega), \quad y_{k} \rightarrow y \text { in } W_{0}^{1, p}(\Omega) .
$$

Then by Lemma 3.2, we have

$$
\lim _{k \rightarrow \infty} \int_{\Omega}\left(\nabla \varphi, \nabla y_{k}\right)_{\mathbb{R}^{N}} u_{k} d x=\int_{\Omega}(\nabla \varphi, \nabla y)_{\mathbb{R}^{N}} u d x \quad \forall \varphi \in C_{0}^{\infty}(\Omega) .
$$

Copyright (C) by SIAM. Unauthorized reproduction of this article is prohibited. 
Now, we show that the limit pair $(u, y)$ is related by inequality $(2.11)$. With that in mind we write down the Minty relation for $\left(u_{k}, y_{k}\right)$ :

$\int_{\Omega}|\nabla \varphi|^{p-2}\left(\nabla \varphi, \nabla \varphi-\nabla y_{k}\right)_{\mathbb{R}^{N}} u_{k} d x+\int_{\Omega} \varphi\left(\varphi-y_{k}\right) d x \geq \int_{\Omega} f\left(\varphi-y_{k}\right) d x \quad \forall \varphi \in C_{0}^{\infty}(\Omega)$.

In view of (3.3) and Lemma 3.2, we can pass to the limit in relation (3.4) as $k \rightarrow \infty$ and arrive at the inequality (2.11) for every $\varphi \in C_{0}^{\infty}(\Omega)$. Finally, from the density of $C_{0}^{\infty}(\Omega)$ in $W_{0}^{1, p}(\Omega),(2.11)$ holds for every $\varphi \in W_{0}^{1, p}(\Omega)$, and hence $y \in W_{0}^{1, p}(\Omega)$ is a solution to the boundary value problem (2.2)-(2.3). This fact together with $u \in \mathfrak{A}_{a d}$ leads us to the conclusion: $(u, y) \in \Xi$.

To conclude this section, we give the existence result for optimal pairs to problem $(2.2)-(2.5)$.

TheOREM 3.5. Let $z_{d} \in L^{2}(\Omega)$ and $f \in L^{2}(\Omega)$ be given functions. Then OCP (P) admits at least one solution.

Proof. Since the set $\Xi$ is nonempty and the cost functional is bounded from below on $\Xi$, it follows that there exists a minimizing sequence $\left\{\left(u_{k}, y_{k}\right)\right\}_{k \in \mathbb{N}} \subset \Xi$ to problem (P), i.e.,

$$
\inf _{(u, y) \in \Xi} I(u, y)=\lim _{k \rightarrow \infty}\left[\int_{\Omega}\left|y_{k}-z_{d}\right|^{2} d x+\int_{\Omega}\left|D u_{k}\right|\right]<+\infty
$$

Hence, $\left\{\left(u_{k}, y_{k}\right)\right\}_{k \in \mathbb{N}}$ is bounded in $B V(\Omega) \times W_{0}^{1, p}(\Omega)$. From Theorem 3.4 we deduce the existence of a subsequence, which we denote in the same way, and a pair $\left(u^{*}, y^{*}\right) \in$ $\Xi$ such that $u_{k} \stackrel{*}{\rightarrow} u$ in $B V(\Omega)$ and $y_{k} \rightarrow y^{*}$ in $W_{0}^{1, p}(\Omega)$. From these convergences we infer that

$$
\lim _{k \rightarrow \infty} \int_{\Omega}\left|y_{k}-z_{d}\right|^{2} d x=\int_{\Omega}\left|y^{*}-z_{d}\right|^{2} d x \text { and } \liminf _{k \rightarrow \infty} \int_{\Omega}\left|D u_{k}\right| \geq \int_{\Omega}\left|D u^{*}\right| \text { by (3.1). }
$$

Thus $I\left(u^{*}, y^{*}\right) \leq \inf _{(u, y) \in \Xi} I(u, y)$ and, consequently, $\left(u^{*}, y^{*}\right)$ is a solution of $(\mathrm{P})$.

4. Regularization of OCP (P). As was pointed out in [12], the $p$-Laplacian $\Delta_{p}(u, y)$ provides an example of a quasi-linear operator in divergence form with a socalled degenerate nonlinearity for $p>2$. In this context we have nondifferentiability of the state $y$ with respect to the control $u$. As follows from Theorem 3.5, this fact is not an obstacle to proving existence of optimal controls in the coefficients, but it causes certain difficulties when deriving the optimality conditions for the considered problem. To overcome this difficulty, we introduce the following family of approximating control problems (see, for comparison, the approach of Casas and Fernández [3] for quasilinear elliptic equations with a distributed control in the right-hand side):

$$
\text { Minimize }\left\{I(u, y)=\int_{\Omega}\left|y-z_{d}\right|^{2} d x+\int_{\Omega}|D u|\right\}
$$

subject to the constraints

$$
\begin{gathered}
-\Delta_{\varepsilon, k, p}(u, y)+y=f \quad \text { in } \Omega \\
y=0 \quad \text { on } \partial \Omega, \\
u \in \mathfrak{A}_{a d}=\left\{v \in B V(\Omega) \mid \xi_{1}(x) \leq v(x) \leq \xi_{2}(x) \text { a.e. in } \Omega\right\} .
\end{gathered}
$$


Here, $k \in \mathbb{N}, \varepsilon$ is a small parameter, which varies within a strictly decreasing sequence of positive numbers converging to 0 , and

$$
\Delta_{\varepsilon, k, p}(u, y)=\operatorname{div}\left(u(x)\left(\varepsilon+\mathcal{F}_{k}\left(|\nabla y|^{2}\right)\right)^{\frac{p-2}{2}} \nabla y\right)
$$

where $\mathcal{F}_{k}: \mathbb{R}_{+} \rightarrow \mathbb{R}_{+}$is a nondecreasing $C^{1}\left(\mathbb{R}_{+}\right)$-function such that

$$
\begin{gathered}
\mathcal{F}_{k}(t)=t \text { if } t \in\left[0, k^{2}\right], \quad \mathcal{F}_{k}(t)=k^{2}+1 \text { if } t>k^{2}+1, \quad \text { and } \\
t \leq \mathcal{F}_{k}(t) \leq t+\delta \text { if } k^{2} \leq t<k^{2}+1 \quad \text { for some } \delta \in(0,1) .
\end{gathered}
$$

As for the function $\mathcal{F}_{k}: \mathbb{R}_{+} \rightarrow \mathbb{R}_{+}$, it can, e.g., be defined by

$$
\mathcal{F}_{k}(t)= \begin{cases}t & \text { if } 0 \leq t \leq k^{2} \\ \left(k^{2}-t\right)^{3}+\left(k^{2}-t\right)^{2}+t & \text { if } k^{2} \leq t \leq k^{2}+1 \\ k^{2}+1 & \text { if } t \geq k^{2}+1\end{cases}
$$

A direct calculation shows that in this case $\delta=4 / 27$.

It is clear that the effect of such perturbations of $\Delta_{p}(u, y)$ is its regularization around critical points where $|\nabla y(x)|$ vanishes or becomes unbounded. In particular, if $y \in W_{0}^{1, p}(\Omega)$ and $\Omega_{k}(y):=\left\{x \in \Omega:|\nabla y(x)|>\sqrt{k^{2}+1}\right\}$, then the chain of inequalities

$$
\begin{aligned}
\left|\Omega_{k}(y)\right| & :=\int_{\Omega_{k}(y)} 1 d x \leq \frac{1}{\sqrt{k^{2}+1}} \int_{\Omega_{k}(y)}|\nabla y(x)| d x \\
& \leq \frac{1}{\sqrt{k^{2}+1}}\left|\Omega_{k}(y)\right|^{\frac{1}{q}}\left(\int_{\Omega}|\nabla y|^{p} d x\right)^{\frac{1}{p}}=\frac{\|y\|_{W_{0}^{1, p}(\Omega)}}{\sqrt{k^{2}+1}}\left|\Omega_{k}(y)\right|^{\frac{p-1}{p}}
\end{aligned}
$$

shows that the Lebesgue measure of the set $\Omega_{k}(y)$ satisfies the estimate

$$
\left|\Omega_{k}(y)\right| \leq\left(\frac{1}{\sqrt{k^{2}+1}}\right)^{p}\|y\|_{W_{0}^{1, p}(\Omega)}^{p} \leq\|y\|_{W_{0}^{1, p}(\Omega)}^{p} k^{-p} \quad \forall y \in W_{0}^{1, p}(\Omega)
$$

i.e., the approximation $\mathcal{F}_{k}\left(|\nabla y|^{2}\right)$ is essential on sets with small Lebesgue measure. The main goal of this section is to show that for each $\varepsilon>0$ and $k \in \mathbb{N}$, the perturbed OCP (4.1)-(4.4) is well posed and its solutions can be considered as a reasonable approximation of optimal pairs to the original problem (2.2)-(2.5). To begin with, we establish a few auxiliary results concerning monotonicity and growth conditions for the regularized $p$-Laplacian $\Delta_{\varepsilon, k, p}$.

For our further analysis, we make use of the following notation:

$$
\|\varphi\|_{\varepsilon, k, u}=\left(\int_{\Omega}\left(\varepsilon+\mathcal{F}_{k}\left(|\nabla \varphi|^{2}\right)\right)^{\frac{p-2}{2}}|\nabla \varphi|^{2} u d x\right)^{1 / p} \quad \forall \varphi \in H_{0}^{1}(\Omega) .
$$

Remark 4.1. For an arbitrary element $y^{*} \in H_{0}^{1}(\Omega)$ let us consider the level set 


$$
\begin{aligned}
\Omega_{k}\left(y^{*}\right):= & \left\{x \in \Omega:\left|\nabla y^{*}(x)\right|>\sqrt{k^{2}+1}\right\} . \text { Then } \\
\left|\Omega_{k}\left(y^{*}\right)\right|: & =\int_{\Omega_{k}\left(y^{*}\right)} 1 d x \leq \frac{1}{\sqrt{k^{2}+1}} \int_{\Omega_{k}\left(y^{*}\right)}\left|\nabla y^{*}(x)\right| d x \\
& \leq \frac{1}{k}\left|\Omega_{k}\left(y^{*}\right)\right|^{\frac{1}{2}}\left(\int_{\Omega_{k}\left(y^{*}\right)}\left|\nabla y^{*}\right|^{2} d x\right)^{\frac{1}{2}} \\
& =\frac{1}{k}\left(\frac{1}{\varepsilon+k^{2}+1}\right)^{\frac{p-2}{4}}\left(\int_{\Omega_{k}\left(y^{*}\right)}\left(\varepsilon+\mathcal{F}_{k}\left(\left|\nabla y^{*}\right|^{2}\right)\right)^{\frac{p-2}{2}}\left|\nabla y^{*}\right|^{2} d x\right)^{\frac{1}{2}}\left|\Omega_{k}\left(y^{*}\right)\right|^{\frac{1}{2}} \\
& \leq \frac{1}{k^{\frac{p}{2}}}\left|\Omega_{k}\left(y^{*}\right)\right|^{\frac{1}{2}} \alpha^{-\frac{1}{2}}\left\|y^{*}\right\|_{\varepsilon, k, u}^{\frac{p}{2}} .
\end{aligned}
$$

Hence, the Lebesgue measure of the set $\Omega_{k}\left(y^{*}\right)$ satisfies the estimate

$$
\left|\Omega_{k}\left(y^{*}\right)\right| \leq \frac{\alpha^{-1}}{k^{p}}\left\|y^{*}\right\|_{\varepsilon, k, u}^{p} \quad \forall y^{*} \in H_{0}^{1}(\Omega) .
$$

Now, we establish the following results.

Proposition 4.2. For every $u \in \mathfrak{A}_{a d}, k \in \mathbb{N}$, and $\varepsilon>0$, the operator

$$
A_{\varepsilon, k, u}:=-\Delta_{\varepsilon, k, p}(u, \cdot)+(\cdot): H_{0}^{1}(\Omega) \rightarrow H^{-1}(\Omega)
$$

is bounded and $\left\|A_{\varepsilon, k, u}\right\| \leq\left(\varepsilon+k^{2}+1\right)^{\frac{p-2}{2}}\left\|\xi_{2}\right\|_{L^{\infty}(\Omega)}+C_{\Omega}^{-2}$.

Proof. From the assumptions on $\mathcal{F}_{k}$ and the boundedness of $u$, we obtain

$$
\begin{aligned}
\left\|A_{\varepsilon, k, u}\right\|= & \sup _{\|y\|_{H_{0}^{1}(\Omega)} \leq 1}\left\|A_{\varepsilon, k, u} y\right\|_{H^{-1}(\Omega)}=\sup _{\|y\|_{H_{0}^{1}(\Omega)} \leq 1\|v\|_{H_{0}^{1}(\Omega)} \leq 1}\left\langle A_{\varepsilon, k, u} y, v\right\rangle_{H^{-1}(\Omega) ; H_{0}^{1}(\Omega)} \\
= & \sup _{\|y\|_{H_{0}^{1}(\Omega)} \leq 1\|v\|_{H_{0}^{1}(\Omega)} \leq 1}\left[\sup _{\Omega}\left(\varepsilon+\mathcal{F}_{k}\left(|\nabla y|^{2}\right)\right)^{\frac{p-2}{2}}(\nabla y, \nabla v)_{\mathbb{R}^{N}} u d x+\int_{\Omega} y v d x\right] \\
\leq & \left(\varepsilon+k^{2}+1\right)^{\frac{p-2}{2}}\left\|\xi_{2}\right\|_{L^{\infty}(\Omega)} \sup _{\|y\|_{H_{0}^{1}(\Omega)} \leq 1\|v\|_{H_{0}^{1}(\Omega)} \leq 1}\|y\|_{H_{0}^{1}(\Omega)}\|v\|_{H_{0}^{1}(\Omega)} \\
& +C_{\Omega}^{-2} \sup _{\|y\|_{H_{0}^{1}(\Omega)} \leq 1\|v\|_{H_{0}^{1}(\Omega)} \leq 1} \sup _{H_{0}^{1}(\Omega)}\|v\|_{H_{0}^{1}(\Omega)} \\
= & \left(\varepsilon+k^{2}+1\right)^{\frac{p-2}{2}}\left\|\xi_{2}\right\|_{L^{\infty}(\Omega)}+C_{\Omega}^{-2},
\end{aligned}
$$

which concludes the proof.

Proposition 4.3. For every $u \in \mathfrak{A}_{a d}, k \in \mathbb{N}$, and $\varepsilon>0$, the operator $A_{\varepsilon, k, u}$ is strictly monotone.

$$
\left(\left(\varepsilon+\mathcal{F}_{k}\left(|a|^{2}\right)\right)^{\frac{p-2}{2}} a-\left(\varepsilon+\mathcal{F}_{k}\left(|b|^{2}\right)\right)^{\frac{p-2}{2}} b, a-b\right)_{\mathbb{R}^{N}} \geq \varepsilon^{\frac{p-2}{2}}|a-b|^{2} \quad \forall a, b \in \mathbb{R}^{N} .
$$

Copyright $@$ ( ) by SIAM. Unauthorized reproduction of this article is prohibited. 
In order to prove it, we note that the left-hand side of (4.9) can be rewritten as

$$
\begin{aligned}
((\varepsilon+ & \left.\left.\mathcal{F}_{k}\left(|a|^{2}\right)\right)^{\frac{p-2}{2}} a-\left(\varepsilon+\mathcal{F}_{k}\left(|b|^{2}\right)\right)^{\frac{p-2}{2}} b, a-b\right)_{\mathbb{R}^{N}} \\
= & \left(\int_{0}^{1} \frac{d}{d s}\left\{\left(\varepsilon+\mathcal{F}_{k}\left(|s a+(1-s) b|^{2}\right)\right)^{\frac{p-2}{2}}(s a+(1-s) b)\right\} d s, a-b\right)_{\mathbb{R}^{N}} \\
= & \int_{0}^{1}\left(\varepsilon+\mathcal{F}_{k}\left(|s a+(1-s) b|^{2}\right)\right)^{\frac{p-2}{2}}|a-b|^{2} d x \\
& +(p-2) \int_{0}^{1}\left\{\left(\varepsilon+\mathcal{F}_{k}\left(|s a+(1-s) b|^{2}\right)\right)^{\frac{p-4}{2}}\right. \\
& \left.\quad \cdot \mathcal{F}_{k}^{\prime}\left(|s a+(1-s) b|^{2}\right)\left|(s a+(1-s) b, a-b)_{\mathbb{R}^{N}}\right|^{2}\right\} d s \\
= & I_{1}+I_{2} .
\end{aligned}
$$

Since $p \geq 2$ and $\mathcal{F}_{k}: \mathbb{R}_{+} \rightarrow \mathbb{R}_{+}$is a nondecreasing $C^{1}\left(\mathbb{R}_{+}\right)$-function, it follows that $I_{2} \geq 0$ for all $a, b \in \mathbb{R}^{N}$. It remains to observe that

$$
\left(\varepsilon+\mathcal{F}_{k}\left(|s a+(1-s) b|^{2}\right)\right) \geq \varepsilon \quad \forall a, b \in \mathbb{R}^{N} .
$$

Hence, $I_{1} \geq \varepsilon^{\frac{p-2}{2}}|a-b|^{2}$ and we arrive at the inequality (4.9). With this we obtain

$$
\begin{aligned}
& \left\langle-\Delta_{\varepsilon, k, p}(u, y)+\Delta_{\varepsilon, k, p}(u, v), y-v\right\rangle_{H^{-1}(\Omega) ; H_{0}^{1}(\Omega)} \\
& =\int_{\Omega} u(x)\left(\left(\varepsilon+\mathcal{F}_{k}\left(|\nabla y|^{2}\right)\right)^{\frac{p-2}{2}} \nabla y-\left(\varepsilon+\mathcal{F}_{k}\left(|\nabla v|^{2}\right)\right)^{\frac{p-2}{2}} \nabla v, \nabla y-\nabla v\right)_{\mathbb{R}^{N}} d x \\
& \geq \alpha \varepsilon^{\frac{p-2}{2}} \int_{\Omega}|\nabla y-\nabla v|^{2} d x=\alpha \varepsilon^{\frac{p-2}{2}}\|y-v\|_{H_{0}^{1}(\Omega)}^{2} \geq 0
\end{aligned}
$$

and

$$
\langle y-v, y-v\rangle_{H^{-1}(\Omega) ; H_{0}^{1}(\Omega)}=\|y-v\|_{L^{2}(\Omega)}^{2} \geq 0 .
$$

Since the relation

$$
\left\langle A_{\varepsilon, k, u} y-A_{\varepsilon, k, u} v, y-v\right\rangle_{H^{-1}(\Omega) ; H_{0}^{1}(\Omega)}=0
$$

implies that $y=v$ almost everywhere in $\Omega$, it follows that the strict monotonicity property (2.8) holds in this case.

Proposition 4.4. For every $u \in \mathfrak{A}_{a d}, k \in \mathbb{N}$, and $\varepsilon>0$, the operator $A_{\varepsilon, k, u}$ is coercive (in the sense of relation (2.10)).

Proof. To check this property it is enough to observe that for any $y \in H_{0}^{1}(\Omega)$, $k \in \mathbb{N}, \varepsilon>0$, and $u \in \mathfrak{A}_{a d}$, we have

$$
\begin{aligned}
& \left\langle A_{\varepsilon, k, u} y, y\right\rangle_{H^{-1}(\Omega) ; H_{0}^{1}(\Omega)}=\left\langle-\Delta_{\varepsilon, k, p}(u, y)+y, y\right\rangle_{H^{-1}(\Omega) ; H_{0}^{1}(\Omega)} \\
& =\int_{\Omega} y^{2} d x+\int_{\Omega}\left(\varepsilon+\mathcal{F}_{k}\left(|\nabla y|^{2}\right)\right)^{\frac{p-2}{2}}|\nabla y|^{2} u d x \geq \alpha \varepsilon^{\frac{p-2}{2}}\|y\|_{H_{0}^{1}(\Omega)}^{2} .
\end{aligned}
$$

We are now in a position to apply the abstract theorem on monotone operators (see Theorem 2.1) to the equation $A_{\varepsilon, k, u} y=f$ with $f \in L^{2}(\Omega)$. Closely following the arguments of section 2 , we arrive at the following assertion.

Copyright (c) by SIAM. Unauthorized reproduction of this article is prohibited. 
TheOrem 4.5. For each $\varepsilon>0, k \in \mathbb{N}, u \in \mathfrak{A}_{a d}$, and $f \in L^{2}(\Omega)$, the boundary value problem (4.2)-(4.3) admits a unique weak solution $y_{\varepsilon, k} \in H_{0}^{1}(\Omega)$, i.e., (4.10)

$\int_{\Omega} u\left(\varepsilon+\mathcal{F}_{k}\left(\left|\nabla y_{\varepsilon, k}\right|^{2}\right)\right)^{\frac{p-2}{2}}\left(\nabla y_{\varepsilon, k}, \nabla \varphi\right)_{\mathbb{R}^{N}} d x+\int_{\Omega} y_{\varepsilon, k} \varphi d x=\int_{\Omega} f \varphi d x \quad \forall \varphi \in H_{0}^{1}(\Omega)$ or, equivalently,

$$
\begin{aligned}
\int_{\Omega} u(x)(\varepsilon+ & \left.\mathcal{F}_{k}\left(|\nabla \varphi|^{2}\right)\right)^{\frac{p-2}{2}}\left(\nabla \varphi, \nabla \varphi-\nabla y_{\varepsilon, k}\right)_{\mathbb{R}^{N}} d x \\
& +\int_{\Omega} \varphi\left(\varphi-y_{\varepsilon, k}\right) d x \geq \int_{\Omega} f\left(\varphi-y_{\varepsilon, k}\right) d x \quad \forall \varphi \in C_{0}^{\infty}(\Omega) .
\end{aligned}
$$

Thus, as follows from Theorem 4.5, for every $\varepsilon>0$ and $k \in \mathbb{N}$, the set of admissible pairs to problem (4.1)-(4.4),

(4.12) $\Xi_{\varepsilon, k}=\left\{(u, y) \mid u \in \mathfrak{A}_{a d}, y \in H_{0}^{1}(\Omega),(u, y)\right.$ are related by equality (4.10) $\}$,

is nonempty. In what follows, we will denote the control problem (4.1)-(4.4) by $\left(\mathrm{P}_{\varepsilon, k}\right)$ :

$$
\left(\mathrm{P}_{\varepsilon, k}\right) \min _{(u, y) \in \Xi_{\varepsilon, k}} I(u, y) .
$$

Analogously to problem (P), we can prove the following theorem.

TheOrem 4.6. For every positive value $\varepsilon>0$ and integer $k \in \mathbb{N}$, the $\operatorname{OCP}\left(\mathrm{P}_{\varepsilon, k}\right)$ has at least one solution.

The proof follows the steps of the proof of Theorem 3.5. Indeed, it is immediate to check that $\Xi_{\varepsilon, k}$ is not empty. Then, we can take a minimizing sequence $\left\{\left(u_{i}, y_{i}\right)\right\}_{i \in \mathbb{N}} \subset$ $\Xi_{\varepsilon, k}$. The lower boundedness of $I$ implies the boundedness of $\left\{\left(u_{i}, y_{i}\right)\right\}_{i \in \mathbb{N}}$ in $B V(\Omega) \times$ $H_{0}^{1}(\Omega)$. Then, arguing as in the proof of Theorem 3.4, we deduce the existence of a subsequence, denoted in the same way, and a pair $\left(u^{*}, y^{*}\right) \in \Xi_{\varepsilon, k}$ such that $u_{i} \stackrel{*}{\rightarrow} u^{*}$ in $B V(\Omega)$ and $y_{i} \rightarrow y^{*}$ in $H_{0}^{1}(\Omega)$. Hence, $I\left(u^{*}, y^{*}\right) \leq \liminf _{i \rightarrow \infty} I\left(u_{i}, y_{i}\right)$.

For our further analysis, we need to obtain some appropriate a priori estimates for the weak solutions to problem (4.2)-(4.3). With that in mind, we make use of the following auxiliary results.

Proposition 4.7. Let $u \in \mathfrak{A}_{a d}, k \in \mathbb{N}$, and $\varepsilon>0$ be given. Then, for arbitrary $g \in L^{2}(\Omega)$ and $y \in H_{0}^{1}(\Omega)$, we have

$$
\left|\int_{\Omega} g y d x\right| \leq C_{\Omega}\|g\|_{L^{2}(\Omega)}\left[\alpha^{-\frac{1}{p}}|\Omega|^{\frac{p-2}{2 p}}\|y\|_{\varepsilon, k, u}+\alpha^{-\frac{1}{2}}\|y\|_{\varepsilon, k, u}^{\frac{p}{2}}\right] .
$$

Proof. Let us fix an arbitrary element $y$ of $H_{0}^{1}(\Omega)$. We associate with this element the set $\Omega^{k}(y)$, where $\Omega^{k}(y):=\{x \in \Omega:|\nabla y(x)|>k\}$. Then, by Friedrich's inequality,

$$
\int_{\Omega} g y d x \leq\|g\|_{L^{2}(\Omega)}\|y\|_{L^{2}(\Omega)} \leq C_{\Omega}\|g\|_{L^{2}(\Omega)}\left(\|\nabla y\|_{L^{2}\left(\Omega \backslash \Omega^{k}(y)\right)^{N}}+\|\nabla y\|_{L^{2}\left(\Omega^{k}(y)\right)^{N}}\right) .
$$

Using the fact that

$$
\begin{aligned}
\|\nabla y\|_{L^{2}\left(\Omega \backslash \Omega^{k}(y)\right)^{N}} & \leq|\Omega|^{\frac{p-2}{2 p}}\|\nabla y\|_{L^{p}\left(\Omega \backslash \Omega^{k}(y)\right)^{N}} \\
& \leq|\Omega|^{\frac{p-2}{2 p}}\left(\int_{\Omega \backslash \Omega^{k}(y)}\left(\varepsilon+|\nabla y|^{2}\right)^{\frac{p-2}{2}}|\nabla y|^{2} d x\right)^{\frac{1}{p}}
\end{aligned}
$$

Copyright (c) by SIAM. Unauthorized reproduction of this article is prohibited. 
and

$$
\begin{gathered}
\mathcal{F}_{k}\left(|\nabla y|^{2}\right)=|\nabla y|^{2} \text { a.e. in } \Omega \backslash \Omega^{k}(y) \text { and } \\
k^{2} \leq \mathcal{F}_{k}\left(|\nabla y|^{2}\right) \leq k^{2}+1 \text { a.e. in } \Omega^{k}(y) \quad \forall k \in \mathbb{N},
\end{gathered}
$$

we obtain

$$
\begin{aligned}
\|\nabla y\|_{L^{2}\left(\Omega \backslash \Omega^{k}(y)\right)^{N}} & \leq|\Omega|^{\frac{p-2}{2 p}}\left(\int_{\Omega \backslash \Omega^{k}(y)}\left(\varepsilon+\mathcal{F}_{k}\left(|\nabla y|^{2}\right)\right)^{\frac{p-2}{2}}|\nabla y|^{2} d x\right)^{\frac{1}{p}} \\
& \leq|\Omega|^{\frac{p-2}{2 p}} \alpha^{-\frac{1}{p}}\|y\|_{\varepsilon, k, u} \\
\|\nabla y\|_{L^{2}\left(\Omega^{k}(y)\right)^{N}} & \leq\left(\int_{\Omega^{k}(y)}\left(\varepsilon+\mathcal{F}_{k}\left(|\nabla y|^{2}\right)\right)^{\frac{p-2}{2}}|\nabla y|^{2} d x\right)^{\frac{1}{2}} \leq \alpha^{-\frac{1}{2}}\|y\|_{\varepsilon, k, u}^{\frac{p}{2}} .
\end{aligned}
$$

As a result, inequality (4.13) immediately follows from (4.14)-(4.16). The proof is complete.

DeFINITION 4.8. Let $\left\{u_{\varepsilon, k}\right\}_{\substack{\varepsilon>0 \\ k \in \mathbb{N}}} \subset \mathfrak{A}_{a d}$ be an arbitrary sequence of admissible controls. We say that a two-parametric sequence $\left\{y_{\varepsilon, k}\right\}_{\substack{\varepsilon>0 \\ k \in \mathbb{N}}} \subset H_{0}^{1}(\Omega)$ is bounded with respect to the $\|\cdot\|_{\varepsilon, k, u_{\varepsilon, k}}$-quasi-seminorm if $\sup _{\substack{\varepsilon>0 \\ k \in \mathbb{N}}}\left\|y_{\varepsilon, k}\right\|_{\varepsilon, k, u_{\varepsilon, k}}<+\infty$.

To conclude this section, let us show that for every $u \in \mathfrak{A}_{a d}$ and $f \in L^{2}(\Omega)$, the sequence of weak solutions to the boundary value problem (4.2)-(4.3), i.e., $\left\{y_{\varepsilon, k}=\right.$ $\left.y_{\varepsilon, k}(u, f)\right\}_{\substack{\varepsilon>0 \\ k \in \mathbb{N}}}$, is bounded with respect to the $\|\cdot\|_{\varepsilon, k, u}$-quasi-seminorm in the sense of Definition 4.8.

Indeed, the integral identity (4.10) together with estimate (4.13) (for $g=f$ ) immediately leads us to the relation

$$
\begin{aligned}
\left\|y_{\varepsilon, k}\right\|_{\varepsilon, k, u}^{p} & :=\int_{\Omega}\left(\varepsilon+\mathcal{F}_{k}\left(\left|\nabla y_{\varepsilon, k}\right|^{2}\right)\right)^{\frac{p-2}{2}}\left|\nabla y_{\varepsilon, k}\right|^{2} u d x \\
& \leq \int_{\Omega}\left(\varepsilon+\mathcal{F}_{k}\left(\left|\nabla y_{\varepsilon, k}\right|^{2}\right)\right)^{\frac{p-2}{2}}\left|\nabla y_{\varepsilon, k}\right|^{2} u d x+\int_{\Omega} y_{\varepsilon, k}^{2} d x=\int_{\Omega} f y_{\varepsilon, k} d x \\
& \leq C_{\Omega}\|f\|_{L^{2}(\Omega)}\left[\alpha^{-\frac{1}{p}}|\Omega|^{\frac{p-2}{2 p}}\left\|y_{\varepsilon, k}\right\|_{\varepsilon, k, u}+\alpha^{-\frac{1}{2}}\left\|y_{\varepsilon, k}\right\|_{\varepsilon, k, u}^{\frac{p}{2}}\right] .
\end{aligned}
$$

As a result, it follows from (4.17) that

$$
\left\|y_{\varepsilon, k}\right\|_{\varepsilon, k, u} \leq \max \left\{C_{f}^{\frac{2}{p}}, C_{f}^{\frac{1}{p-1}}\right\} \quad \forall \varepsilon>0, \forall k \in \mathbb{N}, \forall u \in \mathfrak{A}_{a d}
$$

where $C_{f}:=C\|f\|_{L^{2}(\Omega)}=C_{\Omega}\left(\alpha^{-\frac{1}{p}}|\Omega|^{\frac{p-2}{2 p}}+\alpha^{-\frac{1}{2}}\right)\|f\|_{L^{2}(\Omega)}$. Moreover, taking $g=y=$ $y_{\varepsilon, k}$ in (4.13) and using (4.18), we also have

$$
\left\|y_{\varepsilon, k}\right\|_{L^{2}(\Omega)} \leq \max \left\{C^{2}\|f\|_{L^{2}(\Omega)}, C^{\frac{p}{p-1}}\|f\|_{L^{2}(\Omega)}^{\frac{1}{p-1}}\right\} \quad \forall \varepsilon>0, \forall k \in \mathbb{N}, \forall u \in \mathfrak{A}_{a d} .
$$

5. Asymptotic analysis of the approximate $\operatorname{OCP}\left(\mathbf{P}_{\varepsilon, k}\right)$. Our main intention in this section is to show that optimal solutions to the original OCP (P) can be attained (in some sense) by optimal solutions to the approximated problems $\left(\mathrm{P}_{\varepsilon, k}\right)$. With that in mind, we make use of the concept of variational convergence of constrained minimization problems (see [8]) and study the asymptotic behavior of a family of $\mathrm{OCPs}\left(\mathrm{P}_{\varepsilon, k}\right)$ as $\varepsilon \rightarrow 0$ and $k \rightarrow \infty$. We begin with some auxiliary results concerning the weak compactness in $H_{0}^{1}(\Omega)$ of $\|\cdot\|_{\varepsilon, k, u}$-bounded sequences.

Copyright $@$ by SIAM. Unauthorized reproduction of this article is prohibited. 
LEMMA 5.1. Let $\left\{u_{\varepsilon, k}\right\}_{\substack{>>0 \\ k \in \mathbb{N}}} \subset \mathfrak{A}_{a d}$ be an arbitrary sequence of admissible controls with associated states $\left\{y_{\varepsilon, k}\right\}_{\substack{\varepsilon>0 \\ k \in \mathbb{N}}} \subset H_{0}^{1}(\Omega), y_{\varepsilon, k}=y_{\varepsilon, k}\left(u_{\varepsilon, k}\right)$. Then the sequence $\left\{y_{\varepsilon, k}\right\}_{\substack{\varepsilon>0 \\ k \in \mathbb{N}}}$ is bounded in $H_{0}^{1}(\Omega)$. Moreover, each cluster point $y$ of the sequence $\left\{y_{\varepsilon, k}\right\}_{\substack{c>0 \\ k \in \mathbb{N}}}$ with respect to the weak convergence in $H_{0}^{1}(\Omega)$ satisfies $y \in W_{0}^{1, p}(\Omega)$.

Proof. The boundedness in $H_{0}^{1}(\Omega)$ immediately follows from (4.18) and the estimates

$$
\begin{aligned}
& \left\|y_{\varepsilon, k}\right\|_{H_{0}^{1}(\Omega)} \leq\left\|\nabla y_{\varepsilon, k}\right\|_{L^{2}\left(\Omega \backslash \Omega^{k}\left(y_{\varepsilon, k}\right)\right)^{N}}+\left\|\nabla y_{\varepsilon, k}\right\|_{L^{2}\left(\Omega^{k}\left(y_{\varepsilon, k}\right)\right)^{N}} \\
& \text { by (4.15)-(4.16)} \\
& \stackrel{\leq}{\leq} C_{\Omega}\left[\alpha^{-\frac{1}{p}}|\Omega|^{\frac{p-2}{2 p}}\left\|y_{\varepsilon, k}\right\|_{\varepsilon, k, u}+\alpha^{-\frac{1}{2}}\left\|y_{\varepsilon, k}\right\|_{\varepsilon, k, u}^{\frac{p}{2}}\right],
\end{aligned}
$$

where $u \in \mathfrak{A}_{a d}$ is an arbitrary admissible control and $\Omega^{k}\left(y_{\varepsilon, k}\right):=\left\{x \in \Omega:\left|\nabla y_{\varepsilon, k}(x)\right|\right.$ $>k\}$ for each $k \in \mathbb{N}$.

To establish the second part of the theorem, let us take a subsequence $\left\{y_{\varepsilon_{i}, k_{i}}\right\}_{i \in \mathbb{N}}$ of $\left\{y_{\varepsilon, k}\right\}_{\substack{\varepsilon>0 \\ k \in \mathbb{N}}}$ (here, $\varepsilon_{i} \rightarrow 0$ and $k_{i} \rightarrow \infty$ as $i \rightarrow \infty$ ) and a function $y \in H_{0}^{1}(\Omega)$ such that $y_{\varepsilon_{i}, k_{i}} \rightarrow y$ in $H_{0}^{1}(\Omega)$ as $i \rightarrow \infty$. Further, we fix an index $i \in \mathbb{N}$ and associate it with the following set:

$$
B_{i}:=\bigcup_{j=i}^{\infty} \Omega_{k_{j}}\left(y_{\varepsilon_{j}, k_{j}}\right), \quad \text { where } \Omega_{k_{j}}\left(y_{\varepsilon_{j}, k_{j}}\right):=\left\{x \in \Omega:\left|\nabla y_{\varepsilon_{j}, k_{j}}(x)\right|>\sqrt{k_{j}^{2}+1}\right\} .
$$

Due to estimates (4.8) and (4.18), we see that

$$
\begin{aligned}
\left|B_{i}\right| & \leq \alpha^{-1} \sum_{j=i}^{\infty} \frac{1}{k_{j}^{p}}\left\|y_{\varepsilon_{j}, k_{j}}\right\|_{\varepsilon_{j}, k_{j}, u_{\varepsilon_{j}, k_{j}}}^{p} \leq \alpha^{-1} \sup _{j \in \mathbb{N}}\left\|y_{\varepsilon_{j}, k_{j}}\right\|_{\varepsilon_{j}, k_{j}, u_{\varepsilon_{j}, k_{j}}}^{p} \sum_{j=i}^{\infty} \frac{1}{k_{j}^{p}} \\
& \leq \alpha^{-1} \max \left\{C_{f}^{2}, C_{f}^{\frac{p}{p-1}}\right\} \sum_{j=i}^{\infty} \frac{1}{k_{j}^{p}}<+\infty,
\end{aligned}
$$

and, therefore,

$$
\lim _{i \rightarrow \infty}\left|B_{i}\right|=\mathcal{L}^{N}\left(\limsup _{i \rightarrow \infty} B_{i}\right)=0 .
$$

Using (4.18) again, we get

$$
\begin{aligned}
\int_{\Omega \backslash B_{i}}\left|\nabla y_{\varepsilon_{j}, k_{j}}\right|^{p} d x & \leq \int_{\Omega \backslash B_{i}}\left(\varepsilon_{j}+\left|\nabla y_{\varepsilon_{j}, k_{j}}\right|^{2}\right)^{\frac{p-2}{2}}\left|\nabla y_{\varepsilon_{j}, k_{j}}\right|^{2} d x \\
& \leq \alpha^{-1} \int_{\Omega \backslash B_{i}}\left(\varepsilon_{j}+\mathcal{F}_{k_{j}}\left(\left|\nabla y_{\varepsilon_{j}, k_{j}}\right|^{2}\right)\right)^{\frac{p-2}{2}}\left|\nabla y_{\varepsilon_{j}, k_{j}}\right|^{2} u_{\varepsilon_{j}, k_{j}} d x \\
& \leq \alpha^{-1} \max \left\{C_{f}^{2}, C_{f}^{\frac{p}{p-1}}\right\} \quad \forall j \geq i ;
\end{aligned}
$$

hence $\left\{\nabla y_{\varepsilon_{j}, k_{j}}\right\}$ is bounded in $L^{p}\left(\Omega \backslash B_{i}\right)^{N}$. Since, $\nabla y_{\varepsilon_{j}, k_{j}} \rightarrow \nabla y$ in $L^{2}(\Omega)^{N}$, we infer $\chi_{\Omega \backslash B_{j}} \nabla y_{\varepsilon_{j}, k_{j}} \rightarrow \nabla y$ in $L^{p}(\Omega)^{N}$, where $\chi_{\Omega \backslash B_{j}}$ is the characteristic function of the set $\Omega \backslash B_{j}$. Hence, we obtain

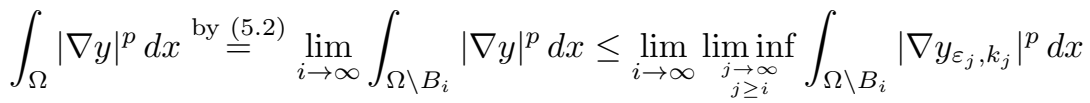

$$
\begin{aligned}
& \stackrel{\text { by (5.3) }}{\leq} \alpha^{-1} \max \left\{C_{f}^{2}, C_{f}^{\frac{p}{p-1}}\right\} \text {. }
\end{aligned}
$$

Copyright $@$ by SIAM. Unauthorized reproduction of this article is prohibited. 
Thus, $y \in W_{0}^{1, p}(\Omega)$, and the proof is complete.

Lemma 5.2. Let $\left\{\varepsilon_{i}\right\}_{i \in \mathbb{N}},\left\{k_{i}\right\}_{i \in \mathbb{N}}$, and $\left\{u_{i}\right\}_{i \in \mathbb{N}} \subset \mathfrak{A}_{a d}$ be sequences such that

$$
\varepsilon_{i} \rightarrow 0, \quad k_{i} \rightarrow \infty, \quad u_{i} \rightarrow u \text { strongly in } L^{1}(\Omega)
$$

Let $y_{i}=y_{\varepsilon_{i}, k_{i}}\left(u_{i}\right)$ and $y=y(u)$ be the solutions of (4.3)-(4.4) and (2.2)-(2.3), respectively. Then

$$
\begin{aligned}
& y_{i} \rightarrow y \text { in } H_{0}^{1}(\Omega) \text { as } i \rightarrow \infty \text {, } \\
& \chi_{\Omega \backslash \Omega_{k}\left(y_{i}\right)} \nabla y_{i} \rightarrow \nabla y \text { strongly in } L^{p}(\Omega)^{N}, \\
& \lim _{i \rightarrow \infty} \int_{\Omega}\left(\varepsilon_{i}+\mathcal{F}_{k_{i}}\left(\left|\nabla y_{i}\right|^{2}\right)\right)^{\frac{p-2}{2}}\left|\nabla y_{i}\right|^{2} u_{i} d x=\int_{\Omega}|\nabla y|^{p} u d x,
\end{aligned}
$$

where $\Omega_{k_{i}}\left(y_{i}\right)$ is defined by (5.1).

Proof. The proof is divided into five steps.

Step 1: $y_{i} \rightarrow y$ in $H_{0}^{1}(\Omega)$. From Lemma 5.1 we deduce the existence of a subsequence, still denoted by $\left\{y_{i}\right\}_{i \in \mathbb{N}} \subset H_{0}^{1}(\Omega)$, and an element $y \in W_{0}^{1, p}(\Omega)$ such that $y_{i} \rightarrow y$ in $H_{0}^{1}(\Omega)$. Let us prove that $y$ is the solution of (2.2)-(2.3). Let us fix an arbitrary test function $\varphi \in C_{0}^{\infty}(\Omega)$ and pass to the limit in the Minty inequality

$$
\begin{aligned}
\int_{\Omega} u_{i}(x)\left(\varepsilon_{i}+\mathcal{F}_{k_{i}}\left(|\nabla \varphi|^{2}\right)\right)^{\frac{p-2}{2}}(\nabla \varphi, \nabla \varphi & \left.-\nabla y_{i}\right)_{\mathbb{R}^{N}} d x \\
& +\int_{\Omega} \varphi\left(\varphi-y_{i}\right) d x \geq \int_{\Omega} f\left(\varphi-y_{i}\right) d x
\end{aligned}
$$

as $i \rightarrow \infty$. Take into account that

$$
\left(\varepsilon_{i}+\mathcal{F}_{k_{i}}\left(|\nabla \varphi|^{2}\right)\right)^{\frac{p-2}{2}} \nabla \varphi \rightarrow|\nabla \varphi|^{p-2} \nabla \varphi \text { strongly in } L^{q}(\Omega)^{N} .
$$

In view of the convergences $\nabla y_{i} \rightarrow \nabla y$ in $L^{2}(\Omega)^{N}$ and $u_{i} \rightarrow u$ strongly in $L^{r}(\Omega)$, for all $r<\infty$, and the boundedness of $\left\{u_{i}\right\}_{i \in \mathbb{N}}$ in $L^{\infty}(\Omega)$, we obtain

$$
\begin{aligned}
& \lim _{i \rightarrow \infty} \int_{\Omega}\left(\varepsilon_{i}+\mathcal{F}_{k_{i}}\left(|\nabla \varphi|^{2}\right)\right)^{\frac{p-2}{2}}(\nabla \varphi, \nabla \varphi)_{\mathbb{R}^{N}} u_{i} d x=\int_{\Omega}|\nabla \varphi|^{p-2}(\nabla \varphi, \nabla \varphi)_{\mathbb{R}^{N}} u d x, \\
& \lim _{i \rightarrow \infty} \int_{\Omega}\left(\varepsilon_{i}+\mathcal{F}_{k_{i}}\left(|\nabla \varphi|^{2}\right)\right)^{\frac{p-2}{2}}\left(\nabla \varphi, \nabla y_{i}\right)_{\mathbb{R}^{N}} u_{i} d x=\int_{\Omega}|\nabla \varphi|^{p-2}(\nabla \varphi, \nabla y)_{\mathbb{R}^{N}} u d x .
\end{aligned}
$$

Thus, passing to the limit in relation (5.8) as $n \rightarrow \infty$, we arrive at the inequality (2.11) for every $\varphi \in C_{0}^{\infty}(\Omega)$. Finally, from the density of $C_{0}^{\infty}(\Omega)$ in $W_{0}^{1, p}(\Omega)$, we infer that (2.11) holds for every $\varphi \in W_{0}^{1, p}(\Omega)$, and hence $y \in W_{0}^{1, p}(\Omega)$ is the solution to the boundary value problem (2.2)-(2.3). Since the solution of $(2.2)-(2.3)$ is unique, the whole sequence $\left\{y_{i}\right\}_{i \in \mathbb{N}}$ converges weakly to $y=y(u)$ in $H_{0}^{1}(\Omega)$.

Step 2: $\chi_{\Omega \backslash \Omega_{k}\left(y_{i}\right)} \nabla y_{i} \rightarrow \nabla y$ in $L^{p}(\Omega)^{N}$. Following the definition of the sets $\Omega_{k_{i}}\left(y_{i}\right)$ and using (4.18), we obtain

$$
\begin{aligned}
\int_{\Omega}\left|\chi_{\Omega \backslash \Omega_{k_{i}}\left(y_{i}\right)} \nabla y_{i}\right|^{p} d x & =\int_{\Omega \backslash \Omega_{k_{i}}\left(y_{i}\right)}\left|\nabla y_{i}\right|^{p} d x \\
& \leq \alpha^{-1} \int_{\Omega \backslash \Omega_{k_{i}}\left(y_{i}\right)}\left(\varepsilon_{i}+\mathcal{F}_{k_{i}}\left(\left|\nabla y_{i}\right|^{2}\right)\right)^{\frac{p-2}{2}}\left|\nabla y_{i}\right|^{2} u_{i} d x \\
& \leq \alpha^{-1}\left\|y_{i}\right\|_{\varepsilon_{i}, k_{i}, u_{i}} \leq C<+\infty \quad \forall i \in \mathbb{N} .
\end{aligned}
$$

Copyright $\odot$ by SIAM. Unauthorized reproduction of this article is prohibited. 
Hence, taking a new subsequence if necessary, we infer the existence of a vectorvalued function $g \in L^{p}(\Omega)^{N}$ such that $\chi_{\Omega \backslash \Omega_{k_{i}}\left(y_{i}\right)} \nabla y_{i} \rightarrow g$ in $L^{p}(\Omega)^{N}$ as $i \rightarrow \infty$. Since $u_{i} \rightarrow u$ in $L^{q}(\Omega)$, we conclude that

$$
\lim _{i \rightarrow \infty} \int_{\Omega \backslash \Omega_{k_{i}}\left(y_{i}\right)}\left(\nabla y_{i}, \nabla \varphi\right) u_{i} d x=\int_{\Omega}(g, \nabla \varphi) u d x \quad \forall \varphi \in C_{0}^{\infty}(\Omega)
$$

On the other hand, in view of the weak convergence $\nabla y_{i} \rightarrow \nabla y$ in $L^{2}(\Omega)^{N}$,

$$
\begin{aligned}
\int_{\Omega}(\nabla y, \nabla \varphi) u d x & =\lim _{i \rightarrow \infty} \int_{\Omega}\left(\nabla y_{i}, \nabla \varphi\right) u_{i} d x \\
& =\lim _{i \rightarrow \infty} \int_{\Omega \backslash \Omega_{k_{i}}\left(y_{i}\right)}\left(\nabla y_{i}, \nabla \varphi\right) u_{i} d x+\lim _{i \rightarrow \infty} \int_{\Omega_{k_{i}}\left(y_{i}\right)}\left(\nabla y_{i}, \nabla \varphi\right) u_{i} d x
\end{aligned}
$$

Since

$$
\begin{aligned}
& \left|\int_{\Omega_{k_{i}}\left(y_{i}\right)}\left(\nabla y_{i}, \nabla \varphi\right) u_{i} d x\right| \leq\left\|u_{i}\right\|_{L^{\infty}(\Omega)}\|\varphi\|_{C^{1}(\bar{\Omega})} \sqrt{\left|\Omega_{k_{i}}\left(y_{i}\right)\right|}\left(\int_{\Omega_{k_{i}}\left(y_{i}\right)}\left|\nabla y_{i}\right|^{2} d x\right)^{1 / 2} \\
& \leq \frac{\left\|u_{i}\right\|_{L^{\infty}(\Omega)}\|\varphi\|_{C^{1}(\bar{\Omega})}}{\left(\varepsilon_{i}+k_{i}^{2}+1\right)^{\frac{p-2}{4}}} \sqrt{\left|\Omega_{k_{i}}\left(y_{i}\right)\right|}\left\|y_{i}\right\|_{\varepsilon_{i}, k_{i}, u_{i}}^{\frac{p}{2}} \\
& \text { by } \stackrel{(4.8),(4.18)}{\leq}\left\|\xi_{2}\right\|_{L^{\infty}(\Omega)}\|\varphi\|_{C^{1}(\bar{\Omega})} \frac{C}{k_{i}^{p-1}} \rightarrow 0 \text { as } i \rightarrow \infty \text {, }
\end{aligned}
$$

it follows from (5.9) and (5.10) that

$$
\int_{\Omega}(g, \nabla \varphi) u d x=\int_{\Omega}(\nabla y, \nabla \varphi) u d x \quad \forall \varphi \in C_{0}^{\infty}(\Omega) .
$$

Hence, $g=\nabla y$ almost everywhere in $\Omega$, and $\chi_{\Omega \backslash \Omega_{k}\left(y_{i}\right)} \nabla y_{i} \rightarrow \nabla y$ in $L^{p}(\Omega)^{N}$ holds.

Step 3: $\chi_{\Omega \backslash \Omega_{k}\left(y_{i}\right)} \nabla y_{i} \rightarrow \nabla y$ in $L^{p}(\Omega)^{N}$. For each $i \in \mathbb{N}$, we have the energy equalities

$$
\begin{aligned}
\int_{\Omega} u_{i}\left(\varepsilon_{i}+\mathcal{F}_{k_{i}}\left(\left|\nabla y_{i}\right|^{2}\right)\right)^{\frac{p-2}{2}}\left|\nabla y_{i}\right|^{2} d x+\int_{\Omega} y_{i}^{2} d x & =\int_{\Omega} f y_{i} d x \\
\int_{\Omega} u(x)|\nabla y|^{p} d x+\int_{\Omega} y^{2} d x & =\int_{\Omega} f y d x .
\end{aligned}
$$

From (5.11) and the fact that $y_{i} \rightarrow y$ in $H_{0}^{1}(\Omega)$, we deduce

$$
\begin{aligned}
& \lim _{i \rightarrow \infty} \int_{\Omega} u_{i}\left(\varepsilon_{i}+\mathcal{F}_{k_{i}}\left(\left|\nabla y_{i}\right|^{2}\right)\right)^{\frac{p-2}{2}}\left|\nabla y_{i}\right|^{2} d x=\lim _{i \rightarrow \infty}\left[\int_{\Omega} f y_{i} d x-\int_{\Omega} y_{i}^{2} d x\right]
\end{aligned}
$$

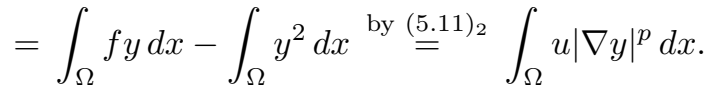

Copyright $@$ by SIAM. Unauthorized reproduction of this article is prohibited. 
Moreover, we have

$$
\begin{aligned}
& \int_{\Omega} u|\nabla y|^{p} d x=\lim _{i \rightarrow \infty} \int_{\Omega}\left(\varepsilon_{i}+\mathcal{F}_{k_{i}}\left(\left|\nabla y_{i}\right|^{2}\right)\right)^{\frac{p-2}{2}}\left|\nabla y_{i}\right|^{2} u_{i} d x \\
& \geq \limsup _{i \rightarrow \infty} \int_{\Omega \backslash \Omega_{k_{i}\left(y_{i}\right)}}\left(\varepsilon_{i}+\mathcal{F}_{k_{i}}\left(\left|\nabla y_{i}\right|^{2}\right)\right)^{\frac{p-2}{2}}\left|\nabla y_{i}\right|^{2} u_{i} d x \\
& \quad \stackrel{(4.6)}{\geq} \limsup _{i \rightarrow \infty} \int_{\Omega \backslash \Omega_{k_{i}}\left(y_{i}\right)}\left(\varepsilon_{i}+\left|\nabla y_{i}\right|^{2}\right)^{\frac{p-2}{2}}\left|\nabla y_{i}\right|^{2} u_{i} d x \\
& \geq \limsup _{i \rightarrow \infty} \int_{\Omega} \chi_{\Omega \backslash \Omega_{k_{i}}\left(y_{i}\right)}\left|\nabla y_{i}\right|^{p} u_{i} d x \geq \liminf _{i \rightarrow \infty} \int_{\Omega} \chi_{\Omega \backslash \Omega_{k_{i}}\left(y_{i}\right)}\left|\nabla y_{i}\right|^{p} u_{i} d x .
\end{aligned}
$$

Since $u_{i} \rightarrow u$ in $L^{r}(\Omega)$ for every $1 \leq r<+\infty,\left\{u_{i}\right\}_{i}$ is bounded in $L^{\infty}(\Omega)$, and $u_{i}(x) \geq \alpha$ for almost all $x \in \Omega$, it is easy to check that $\chi_{\Omega \backslash \Omega_{k_{i}}\left(y_{i}\right)} \nabla y_{i} u_{i}^{1 / p} \rightarrow \nabla y u^{1 / p}$ in $L^{p}(\Omega)^{N}$. Using this convergence and (5.13), we get

$$
\begin{aligned}
& \int_{\Omega} u|\nabla y|^{p} d x \geq \limsup _{i \rightarrow \infty} \int_{\Omega} u_{i} \chi_{\Omega \backslash \Omega_{k_{i}}\left(y_{i}\right)}\left|\nabla y_{i}\right|^{p} d x \\
& \geq \liminf _{i \rightarrow \infty} \int_{\Omega} u_{i} \chi_{\Omega \backslash \Omega_{k_{i}}\left(y_{i}\right)}\left|\nabla y_{i}\right|^{p} d x=\liminf _{i \rightarrow \infty}\left\|\chi_{\Omega \backslash \Omega_{k_{i}}\left(y_{i}\right)} \nabla y_{i} u_{i}^{1 / p}\right\|_{L^{p}(\Omega)^{N}}^{p} \\
& \geq\left\|\nabla y u^{1 / p}\right\|_{L^{p}(\Omega)^{N}}^{p}=\int_{\Omega} u|\nabla y|^{p} d x .
\end{aligned}
$$

The weak convergence $\chi_{\Omega \backslash \Omega_{k_{i}}\left(y_{i}\right)} \nabla y_{i} u_{i}^{1 / p} \rightarrow \nabla y u^{1 / p}$ in $L^{p}(\Omega)^{N}$ and the convergence of their norms $\left\|\chi_{\Omega \backslash \Omega_{k_{i}}\left(y_{i}\right)} \nabla y_{i} u_{i}^{1 / p}\right\|_{L^{p}(\Omega)^{N}} \rightarrow\left\|\nabla y u^{1 / p}\right\|_{L^{p}(\Omega)^{N}}$ imply the strong convergence $\chi_{\Omega \backslash \Omega_{k_{i}}\left(y_{i}\right)} \nabla y_{i} u_{i}^{1 / p} \rightarrow \nabla y u^{1 / p}$ in $L^{p}(\Omega)^{N}$. Now, it is a simple exercise to check the strong convergence $\chi_{\Omega \backslash \Omega_{k_{i}}\left(y_{i}\right)} \nabla y_{i} \rightarrow \nabla y$ in $L^{p}(\Omega)^{N}$.

Step 4: Proof of (5.7). From (5.6) and (5.13) we obtain

$$
\lim _{i \rightarrow \infty} \int_{\Omega_{k_{i}}\left(y_{i}\right)}\left(\varepsilon_{i}+\mathcal{F}_{k_{i}}\left(\left|\nabla y_{i}\right|^{2}\right)\right)^{\frac{p-2}{2}}\left|\nabla y_{i}\right|^{2} u_{i} d x=0 .
$$

Let us prove that

$$
\lim _{i \rightarrow \infty} \int_{\Omega \backslash \Omega_{k_{i}}\left(y_{i}\right)}\left(\varepsilon_{i}+\mathcal{F}_{k_{i}}\left(\left|\nabla y_{i}\right|^{2}\right)\right)^{\frac{p-2}{2}}\left|\nabla y_{i}\right|^{2} u_{i} d x=\int_{\Omega}|\nabla y|^{p} u d x .
$$

This is established as follows. From (4.6) we deduce

$$
\begin{aligned}
\left(\varepsilon_{i}+\mathcal{F}_{k_{i}}\left(\left|\nabla y_{i}\right|^{2}\right)\right)^{\frac{p-2}{2}} & \left|\nabla y_{i}\right|^{2} \chi_{\Omega \backslash \Omega_{k_{i}}\left(y_{i}\right)} \\
& \leq\left(\varepsilon_{i}+\delta+\left|\nabla y_{i}\right|^{2}\right)^{\frac{p-2}{2}}\left|\nabla y_{i}\right|^{2} \chi_{\Omega \backslash \Omega_{k_{i}}\left(y_{i}\right)} \\
& \leq 2^{\frac{p-2}{2}}\left(\left(\varepsilon_{i}+\delta\right)^{\frac{p-2}{2}}\left|\nabla y_{i}\right|^{2}+\left|\nabla y_{i}\right|^{p}\right) \chi_{\Omega \backslash \Omega_{k_{i}}\left(y_{i}\right)} .
\end{aligned}
$$

From (5.6) we know that the last term converges in $L^{1}(\Omega)$. Taking a subsequence if necessary, we can dominate it by an $L^{1}(\Omega)$ function. Then by a simple application of Lebesgue's dominated convergence theorem we deduce (5.15). Finally, (5.14) and (5.15) imply (5.7).

Copyright $\odot$ by SIAM. Unauthorized reproduction of this article is prohibited. 
Step 5: $y_{i} \rightarrow y$ in $H_{0}^{1}(\Omega)$. First, we apply (5.14) to deduce

$$
\lim _{i \rightarrow \infty} \int_{\Omega_{k}\left(y_{i}\right)}\left|\nabla y_{i}\right|^{2} d x \leq \frac{1}{\alpha} \lim _{i \rightarrow \infty} \int_{\Omega_{k}\left(y_{i}\right)}\left(\varepsilon_{i}+\mathcal{F}_{k}\left(\left|\nabla y_{i}\right|^{2}\right)\right)^{\frac{p-2}{2}}\left|\nabla y_{i}\right|^{2} u_{i} d x=0 .
$$

Now, combining this estimate and (5.6) we conclude that

$$
\nabla y_{i}=\chi_{\Omega_{k}\left(y_{i}\right)} \nabla y_{i}+\chi_{\Omega \backslash \Omega_{k}\left(y_{i}\right)} \nabla y_{i} \rightarrow \nabla y \text { strongly in } L^{2}(\Omega)^{N} .
$$

This completes the proof.

We are now in a position to show that optimal pairs to the approximated OCP $\left(\mathrm{P}_{\varepsilon, k}\right)$ lead in the limit to optimal solutions of the original OCP $(\mathrm{P})$.

TheOREM 5.3. Let $\left\{\left(u_{\varepsilon, k}^{0}, y_{\varepsilon, k}^{0}\right)\right\}_{\substack{\varepsilon>0 \\ k \in \mathbb{N}}}$ be an arbitrary sequence of optimal pairs to the perturbed problems $\left(\mathrm{P}_{\varepsilon, k}\right)$. Then, this sequence is bounded in $B V(\Omega) \times H_{0}^{1}(\Omega)$, and any cluster point $\left(u^{0}, y^{0}\right)$ with respect to the (weak-* weak) topology is a solution of the $\operatorname{OCP}(\mathrm{P})$. Moreover, if for one subsequence we have $u_{\varepsilon, k}^{0} \stackrel{*}{\rightarrow} u^{0}$ in $B V(\Omega)$ and $y_{\varepsilon, k}^{0} \rightarrow y^{0}$ in $H_{0}^{1}(\Omega)$, then the following properties hold:

$$
\begin{aligned}
& \lim _{\substack{\varepsilon \rightarrow 0 \\
k \rightarrow \infty}}\left(u_{\varepsilon, k}^{0}, y_{\varepsilon, k}^{0}\right)=\left(u^{0}, y^{0}\right) \text { strongly in } L^{1}(\Omega) \times H_{0}^{1}(\Omega), \\
& \lim _{\substack{\varepsilon \rightarrow 0 \\
k \rightarrow \infty}} \int_{\Omega}\left|D u_{\varepsilon, k}^{0}\right|=\int_{\Omega}\left|D u^{0}\right|, \\
& \lim _{\substack{\varepsilon \rightarrow 0 \\
k \rightarrow \infty}} \chi_{\Omega \backslash \Omega_{k}\left(y_{\varepsilon, k}^{0}\right)} \nabla y_{\varepsilon, k}^{0}=\nabla y^{0} \quad \text { strongly in } L^{p}(\Omega)^{N}, \\
& \lim _{\substack{\varepsilon \rightarrow 0 \\
k \rightarrow \infty}} \int_{\Omega}\left(\varepsilon+\mathcal{F}_{k}\left(\left|\nabla y_{\varepsilon, k}^{0}\right|^{2}\right)\right)^{\frac{p-2}{2}}\left|\nabla y_{\varepsilon, k}^{0}\right|^{2} u_{\varepsilon, k}^{0} d x=\int_{\Omega}\left|\nabla y^{0}\right|^{p} u^{0} d x, \\
& \lim _{\substack{\varepsilon \rightarrow 0 \\
k \rightarrow \infty}} I\left(u_{\varepsilon, k}^{0}, y_{\varepsilon, k}^{0}\right)=I\left(u^{0}, y^{0}\right) .
\end{aligned}
$$

Proof. The boundedness of $\left\{\left(u_{\varepsilon, k}^{0}, y_{\varepsilon, k}^{0}\right)\right\}_{\substack{\varepsilon>0 \\ k \in \mathbb{N}}}$ in $B V(\Omega) \times H_{0}^{1}(\Omega)$ is an immediate consequence of the boundedness of $\mathfrak{A}_{a d}$ in $B V(\Omega)$ and Lemma 5.1. Let us take a subsequence, denoted in the same way, such that $u_{\varepsilon, k}^{0} \stackrel{*}{\rightarrow} u^{0}$ in $B V(\Omega)$ and $y_{\varepsilon, k}^{0} \rightarrow y^{0}$ in $H_{0}^{1}(\Omega)$. From Remark 3.1 we get that

$$
\lim _{\substack{\varepsilon \rightarrow 0 \\ k \rightarrow \infty}} u_{\varepsilon, k}^{0}=u^{0} \text { strongly in } L^{1}(\Omega) \text { and } \int_{\Omega}\left|D u^{0}\right| \leq \liminf _{\substack{\varepsilon \rightarrow 0 \\ k \rightarrow \infty}} \int_{\Omega}\left|D u_{\varepsilon, k}^{0}\right| .
$$

From these convergence properties we infer that $u^{0} \in \mathfrak{A}_{a d}$. Moreover, Lemma 5.2 implies that $y^{0}$ is the solution of $(2.2)-(2.3)$ corresponding to $u=u^{0}$; therefore $\left(u^{0}, y^{0}\right) \in \Xi$. Combining (5.5) and (5.21), we deduce (5.16). Convergences (5.18) and (5.19) follow from (5.6) and (5.7). Let us prove that $\left(u^{0}, y^{0}\right)$ is a solution of $(\mathrm{P})$. Given an arbitrary element $(u, y) \in \Xi$, we define $u_{\varepsilon, k}=u$ and $y_{\varepsilon, k}$ as the solution of (4.2)-(4.3); hence $\left(u_{\varepsilon, k}, y_{\varepsilon, k}\right) \in \Xi_{\varepsilon, k}$. From (5.5) and (5.7) we get

$$
I(u, y)=\lim _{\substack{\varepsilon \rightarrow 0 \\ k \rightarrow \infty}} I\left(u, y_{\varepsilon, k}\right)=\lim _{\substack{\varepsilon \rightarrow 0 \\ k \rightarrow \infty}} I\left(u_{\varepsilon, k}, y_{\varepsilon, k}\right) .
$$

Now, using $(5.5),(5.16),(5.21)$, the above identity, and the fact that $\left(u_{\varepsilon, k}^{0}, y_{\varepsilon, k}^{0}\right)$ is a solution of $\left(\mathrm{P}_{\varepsilon, k}\right)$, we get

$$
I\left(u^{0}, y^{0}\right) \leq \liminf _{\substack{\varepsilon \rightarrow 0 \\ k \rightarrow \infty}} I\left(u_{\varepsilon, k}^{0}, y_{\varepsilon, k}^{0}\right) \leq \limsup _{\substack{\varepsilon \rightarrow 0 \\ k \rightarrow \infty}} I\left(u_{\varepsilon, k}^{0}, y_{\varepsilon, k}^{0}\right) \leq \limsup _{\substack{\varepsilon \rightarrow 0 \\ k \rightarrow \infty}} I\left(u_{\varepsilon, k}, y_{\varepsilon, k}\right)=I(u, y) .
$$

Copyright $@$ by SIAM. Unauthorized reproduction of this article is prohibited. 
Since $(u, y)$ is arbitrary in $\Xi$, this implies that $\left(u^{0}, y^{0}\right)$ is a solution of $(\mathrm{P})$. Moreover, taking $(u, y)=\left(u^{0}, y^{0}\right)$ in the above inequalities, (5.20) is proved. Finally, (5.17) is an immediate consequence of (5.20) and the convergence properties established previously.

Since Theorem 5.3 does not give an answer to whether the entire set of solutions $\Xi^{o p t}$ to problem (2.2)-(2.5) can be attained in such a way, the following result sheds some light on this matter.

Corollary 5.4. Let $\left(u^{0}, y^{0}\right) \in \Xi^{\text {opt }}$ be an optimal solution to the OCP $(\mathrm{P})$ such that there is a closed neighborhood $\mathcal{U}\left(u^{0}\right)$ of $u^{0}$ in the norm topology of $L^{1}(\Omega)$ that satisfies

$$
I\left(u^{0}, y^{0}\right)<I(u, y) \quad \forall u \in \mathfrak{A}_{a d} \cap \mathcal{U}\left(u^{0}\right) \text { such that }(u, y) \in \Xi \text { and } u \neq u^{0} .
$$

Then there exists a sequence of local minima $\left(u_{\varepsilon, k}^{0}, y_{\varepsilon, k}^{0}\right)$ of problems $\left(\mathrm{P}_{\varepsilon, k}\right)$ such that

$$
\left(u_{\varepsilon, k}^{0}, y_{\varepsilon, k}^{0}\right) \rightarrow\left(u^{0}, y^{0}\right) \quad \text { in the sense of Theorem 5.3. }
$$

Proof. By the strict local optimality of $\left(u^{0}, y^{0}\right)$, we have that it is the unique solution of

$$
\text { (Q) } \min _{(u, y) \in \Xi, u \in \mathcal{U}\left(u^{0}\right)} I(u, y) .
$$

For every $\varepsilon$ and $k$ let us consider the control problems

$$
\left(\mathrm{Q}_{\varepsilon, k}\right) \min _{(u, y) \in \Xi_{\varepsilon, k}, u \in \mathcal{U}\left(u^{0}\right)} I(u, y) .
$$

Since $\left(u^{0}, y_{\varepsilon, k}\left(u^{0}\right)\right) \in \Xi_{\varepsilon, k}$, it follows that $\left(\mathrm{Q}_{\varepsilon, k}\right)$ has feasible controls; hence there exists at least one solution $\left(u_{\varepsilon, k}^{0}, y_{\varepsilon, k}^{0}\right)$ of $\left(\mathrm{Q}_{\varepsilon, k}\right)$ for every $(\varepsilon, k)$. Now, arguing as in the proof of Theorem 5.3, we deduce that $\left(u_{\varepsilon, k}^{0}, y_{\varepsilon, k}^{0}\right) \rightarrow\left(\tilde{u}^{0}, \tilde{y}^{0}\right)$ strongly in $L^{1}(\Omega) \times H_{0}^{1}(\Omega)$, and $\left(\tilde{u}^{0}, \tilde{u}^{0}\right)$ is a solution of $(\mathrm{Q})$. Since $\left(u^{0}, y^{0}\right)$ is the unique solution of $(\mathrm{Q})$, we infer that $\left(u_{\varepsilon, k}^{0}, y_{\varepsilon, k}^{0}\right) \rightarrow\left(\tilde{u}^{0}, \tilde{y}^{0}\right)$ strongly in $L^{1}(\Omega) \times H_{0}^{1}(\Omega)$. This implies the existence of $\varepsilon^{0}$ and $k^{0}$ such that $u_{\varepsilon, k}^{0}$ belongs to the interior of $\mathcal{U}\left(u^{0}\right)$ for every $\varepsilon \leq \varepsilon^{0}$ and $k \geq k^{0}$. Consequently, $\left(u_{\varepsilon, k}^{0}, y_{\varepsilon, k}^{0}\right)$ is a local minimum of $\left(\mathrm{P}_{\varepsilon, k}\right)$ for every $\varepsilon \leq \varepsilon^{0}$ and $k \geq k^{0}$. This concludes the proof.

\section{REFERENCES}

[1] L. Ambrosio, N. Fusco, and D. Pallara, Functions of Bounded Variation and Free Discontinuity Problems, The Clarendon Press, Oxford University Press, New York, 2000.

[2] E. CASAS, Optimal control in coefficients of elliptic equations with state constraints, Appl. Math. Optim., 26 (1992), pp. 21-37, http://dx.doi.org/10.1007/BF01218394.

[3] E. CASAs And L. A. Fernández, Optimal control of quasilinear elliptic equations with non differentiable coefficients at the origin, Rev. Mat. Univ. Complut. Madrid, 4 (1991), pp. $227-250$.

[4] M. R. Dostanić, On an inequality of Friedrich's type, Proc. Amer. Math. Soc., 125 (1997), pp. 2115-2118.

[5] I. Ekeland and R. Temam, Convex Analysis and Variational Problems, North-Holland, Amsterdam, Oxford; Elsevier, New York, 1976.

[6] E. Giusti, Minimal Surfaces and Functions of Bounded Variation, Birkhäuser Boston, Boston, 1984.

[7] D. Kinderlehrer and G. Stampacchia, An Introduction to Variational Inequalities and Their Applications, Academic Press, New York, 1980.

[8] P. I. Kogut and G. R. Leugering, Optimal Control Problems for Partial Differential Equations on Reticulated Domains. Approximation and Asymptotic Analysis, Systems Control Found. Appl., Birkhäuser Verlag, Basel, 2011. 
[9] J.-L. Lions, Some Methods of Solving Nonlinear Boundary Value Problems, Dunod-GauthierVillars, Paris, 1969.

[10] F. Murat, Un contre-exemple pour le problème du contrôle dans les coefficients. C. R. Acad. Sci. Paris Sér. A-B, 273 (1971), pp. A708-A711.

[11] F. Murat, Contre-examples pour divers problèmes où le contrôle intervient dans les coefficients, Ann. Mat. Pura Appl. (4), 112 (1977), pp. 49-68, http://dx.doi.org/10.1007/ BF02413475.

[12] T. RoubíčEK, Nonlinear Partial Differential Equations with Applications, Birkhäuser Verlag, Basel, 2013.

[13] R. E. ShowAlter, Monotone Operators in Banach Space and Nonlinear Partial Differential Equations, American Mathematical Society, Providence, RI, 1997.

[14] L. TARTAR, Problèmes de contrôle des coefficients dans des équations aux dérivées partielles, in Control Theory, Numerical Methods and Computer Systems Modelling, Lecture Notes in Econom. and Math. Systems 107, A. Bensoussan and J. L. Lions, eds., Springer-Verlag, Berlin, 1975. 